\title{
The development of semantic reading among students of a defectological profile in the digitalization of education
}

\author{
Natalia Kalugina ${ }^{1}$, Elena Larina $^{2, *}$, Natalia Garkusha ${ }^{2}$, and Diana Kuznetsova ${ }^{3}$ \\ ${ }^{1}$ Far Eastern Federal University, 690922, i. Russian, s. Ajax, 10, Vladivostok, Russia \\ ${ }^{2}$ Pacific State University Pedagogical Institute, str. Karl-Marx, 68, 680000 Khabarovsk, Russia \\ ${ }^{3}$ Khabarovsk Institute for the Development of Education, str. Transbaikal, 10, 68000 Khabarovsk, \\ Russia
}

\begin{abstract}
The study of psychological and pedagogical conditions, and the formation of professionally significant qualities of the future speech therapist, is associated with the formation of intonational and semantic skills, which affects the training of future specialists. The presented forms, methods of organizing the educational process on the example of specific subjects of the educational plan, made it possible to increase the competenc e of students. Specially selected exercises during the "Pedagogy" discipline were focused on mastering the scientific style, which contributed to the development of semantic reading. The results of the work done confirm that if specially selected tasks are included in the educational process, this allows for better training of future speech therapists in accordance with the standard of professional training in the context of digitalization.
\end{abstract}

\section{Introduction}

The current stage of development causes the need to reform education, changes the requirements for the quality of training of future specialists. Digital automation of education will lead to a reorganization of the learning process, to its globalization, since it involves gaining access to wide information educational resources. However, not all professional competencies can be transformed into training in working with the latest information technologies. General cultural and general educational competences do not lose their relevance, especially for students of a defectological profile. Such as, the willingness to improve their speech culture, the ability to communicate in oral and written forms, the ability to self-education and social and professional mobility, and private, the ability to understand a scientific text and correctly reproduce it intonationally, to use a scientific style of presentation when performing experimental research [1].

The task of this article was to describe the methods of organizing the educational process on the example of mastering the scientific discipline "Pedagogy", which made it possible to increase the competence and competence of students with a defectological profile, namely, the formation of methods of a semantic and scientific style of presentation. 
At the same time, it is difficult to separate the mutual influence of semantic and intonationa l-expressive reading on the development of the student's personality as a whole. This moment is noted by both foreign authors and Russian scientists in the field of psychology, pedagogy and defectology. Marcos Mendes drew attention to the peculiarities of mastering semantic reading when organizing scientific activities among students. Scientific literature, according to the author, is growing at an ever faster pace. Scientists find it difficult to navigate the vast amount of relevant literature. This overload can potentially lead to increasingly selective or superficial reading. The consequences are detrimental to the training of new scientists, lowering quality standards. Good reading skills are essential and must be instilled from the outset of a scientific career. Good reading skills are essential at all stages of a scientific career. Scholars should develop them, love literature, and instill these skills in their students as part of their mentoring. Thus, they can play an important role in the mentoring process as a way to set an example and as a tool for sharing knowledge and curiosity. Good self-discipline is at the core of good reading skills. Since self-discipline is an essential skill for aspiring scholars, they should not underestimate reading as a way to develop this skill. [2].

Russian scientists pointed out that understanding the text is associated with the disclosure of the hierarchy of semantic connections of the perceived speech message $[3,4]$. It is known that various components of a speech utterance constitute a hierarchically organized complex unity (S.L. Rubinstein) [5]. At the same time, all stages of semantic perception are not noticed by a person, they take place at an unconscious level. (Z.I. Klychnikova) [6]. Reading is always subordinated to the main task - understanding the written message. It is always a two-way technical and semantic process. The technical side of reading covers the components of the reading skill such as way of reading, correctness, expressiveness, speed or pace. The semantic side of reading is understood as a kind of cumulative awareness when reading such aspects as the meaning of words (direct and figurative), a combination of words with semantic connections in enlarged parts (sentence, paragraph, chapter), a holistic perception of the main meaning of the entire content.

It is important that during the professional training of future specialists at the university, these two areas in the development of reading skills are taken into account, students mastered new language competencies and implemented them in their activities, taking into account the influence of modern trends - digitalization of education.

M. Kushnir defines digitalization in the educational environment as an open digital environment created to provide a set of tasks for the educational process. "Open" means the ability and right of any user to use different information systems, replace them or add new ones. For this, conditions and publicly published rules must be provided. The "environment" is fundamentally different from the system in that it includes completely different elements: both coordinated with each other and duplicating, competing and even antagonistic. This allows the environment to develop more dynamically. The system, in contrast to the environment, is created for specific purposes and in a coordinated unity. It is determined by the range of compliance with the real external conditions provided for in the project initially. The faster conditions change, the shorter the life of the systems. Rajiv Sivaraman defines digitalization as the transition to a digital way of communication. E. L. Vartanova, S. S. Smirnov see this concept as the translation of information into digital. A. Marey points out that digitalization implies the need to improve the quality of human perception of information and work with it (especially at the scientific level) [7]. Modern information and digital technologies provide students with access to information sources, increase the efficiency of independent work, introduce the subject world and contribute to the development of information competence [8]. 


\section{Research materials and methods}

Research object: development of semantic reading among students of a defectological profil e. The choice of the problematic area of research is due to a number of contradictions: 1) the need of general education institutions for speech therapists with communicative compet ence, and insufficient development of this competence in the higher education system; 2) a low level of speech culture of students and a large number of interactive means to the detriment of the necessary means of oral communication; 3) the trend of introducing digitalization into the system of higher education and determining the necessary share of its application in the organization of purposeful theoretical and practical teaching of semantic reading.

Research objectives: 1) to determine the directions of the structural and meaningful met hodological technology of teaching students of a defectological profile to semantic reading; 2) to include in the educational process in the content of the scientific discipline "Pedagogy " methods of mastering the understanding of the text and the development of the scientific style of presentation.

In order to check the initial positions, a number of research methods were used: method s of empirical analysis: analysis of the products of students' activity when performing speci ally selected tasks, a comparative analysis of the understanding of a scientific text among students at the stages of learning.

The analysis of the products of activity was carried out using the author's standardized forms: the form for the analysis of products of activity was aimed at diagnosing the levels of development of semantic reading. Each form included three assessment criteria, three levels of development of each criterion, as well as skills, acting as units of analysis, corresponding to each level (5-6 for each level). Achieving the objectivity of the assessment procedure, the analysis of the products of activity was independently assessed by each of the four experts (the authors of this article).

The level of expression of the criterion for each student was determined as follows: 1) the frequency of occurrence of skills (units of analysis) of each level was calculated in the expert forms; 2) then the summation of frequencies was carried out for each level of four expert forms; 3) the final level of development of the respondent's criterion was determined by identifying the highest frequency.

Diagnostics was carried out twice: 1) the ascertaining stage in February 2019 in the group of 2nd year respondents (4th semester); 2) a control stage in December 2019 in the same group of students of the 3rd course (5th semester) after carrying out work on the development of semantic reading. The sample of the study included 26 students of one student group studying in the specialty "Speech therapy". Research base: Pedagogical Institute of the Pacific State University (c. Khabarovsk).

Work on the development of semantic reading took place within the framework of the development of a scientific discipline, which is included in the curriculum for the training of future specialists. During the training, practical skills were developed by performing a variety of exercises focused on the final product. Moreover, it is not the sum of academic knowledge that is considered as a result, but preparation for real pedagogical activity.

\section{Results and its discussion}

Analysis of the products of the development of semantic reading showed progress, which is expressed, first of all, in the development of the scientific style. For analysis, you can cite fragments of the essay "The duty of a teacher-defectologist", which in terms of their content can be divided into three groups: a high level of development of the scientific style of presentation, an average level of development of a scientific style of presentation (closer to 
journalistic) and a low level of development of a scientific style of presentation ( rather household).

A fragment of an essay that can be attributed to a high level of development of the scientific style of presentation: Studying the basics of psychology and pedagogy, I can say that the profession of a teacher-defectologist is of great importance in the modern world. In order to become a good specialist, high intelligence, intuition, observation, and emotional stability are required. A teacher-defectologist should be attentive, responsive, tactful, sociable, patient. You need a competent and intelligible speech, a well-delivered voice.

The average level of development of the scientific style of presentation can be seen in the following fragment: We often think about different things around us, events taking place in the world where adults and children live. We use different words to express our thoughts. But children with disabilities experience difficulties in knowing and talking about this world.

And a fragment of an essay on the same topic, a low level of development of the scientific style of presentation: for me, the duty of a teacher-defectologist, first of all, is responsibility for your business and the people in whom you invest your knowledge. Before taking on any business, a person must realize and understand what he wants from or from him business (punctuation and author's style).

We can assume on the basis of the presented fragments about the relationship between the level of development of semantic reading and the level of proficiency in the scientific style. This connection is directly dependent: the higher the level of semantic reading, the more thoughtful the student's statement, through written speech. The presented analysis shows the possibility of obtaining diagnostic results, which make it possible to speak about the ascertaining of the development of semantic reading and mastery of the scientific style at the ascertaining stage in percentage terms.

\section{The ability to pose questions to a part of the synopsis. \\ The skill of comprehending a scientific concept \\ Scientific writing skills - essays}

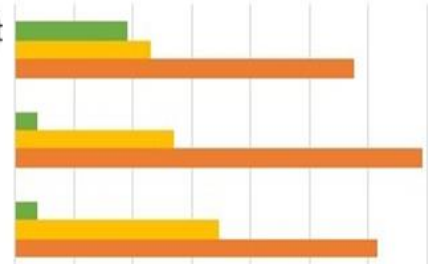

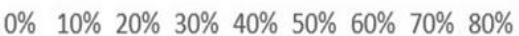

high level of development average level of development

low level of development

Fig. 1. The results of diagnostics of the levels of development of semantic reading among students at the ascertaining stage.

A similar analysis can be done on the basis of differentiation according to the levels of refined concepts of the scientific discipline "Pedagogy" and the ability to pose questions when taking notes of scientific articles. Comparative analysis showed the following results for three types of work: essay, concept formulation, question to a part of the outlined article (Fig. 1)

As we see in the diagram, at the beginning of the academic year, first-year students showed very low results, which determined the need for work on the development of semantic reading. 
This work was carried out during the academic year, in addition to systematic tasks and exercises aimed at developing semantic reading and mastering the scientific style, students took part in a student scientific conference, articles were written under the guidance of a teacher, with an analysis of the results of their scientific research (Fig. 2)

\section{The ability to pose questions to a part of the synopsis \\ The skill of comprehending a scientific concept \\ Scientific writing skills - essays}

\author{
high level of development \\ low level of development
}

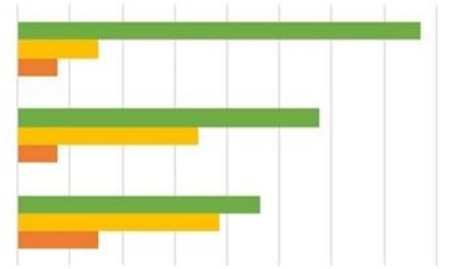

$0 \% 10 \% 20 \% 30 \% 40 \% 50 \% 60 \% 70 \% 80 \% 90 \%$

naverage level of development

Fig. 2. The results of diagnostics of the levels of development of semantic reading among students at the control stage.

A comparative analysis showed that the use of special methods, tasks and exercises increases the level of development of meaningful reading and improves the quality of the educational process, which is very significant in the context of digitalization of education, when work with text is mandatory.

The statistical analysis of the obtained differences, carried out using Fisher's phicriterion, made it possible to confirm the significance of changes in the distribution of the frequencies of developmental levels in each criterion (Table 1). Significant changes were found in the low and high levels of development of semantic reading among students at the ascertaining and control stages.

Table 1. The results of a comparative analysis of diagnostics of the level of development of semantic reading before and after the introduction of special conditions.

\begin{tabular}{|l|l|l|l|}
\hline Level / Criterion & $\begin{array}{l}\text { Scientific Writing } \\
\text { Skills - Essays }\end{array}$ & $\begin{array}{l}\text { Skills of } \\
\text { comprehending a } \\
\text { scientific concept }\end{array}$ & $\begin{array}{l}\text { Ability to pose } \\
\text { questions to a } \\
\text { part of the } \\
\text { synopsis }\end{array}$ \\
\hline Low level of development & $\varphi=3,6 ; p<0,001$ & $\varphi=5,1 ; p<0,001$ & $\varphi=4,2 ; p<0,001$ \\
\hline Average level of development & $\varphi=0,3$ & $\varphi=0,6$ & $\varphi=0,7$ \\
\hline High level of development & $\varphi=3,9 ; p<0,001$ & $\varphi=4,8 ; p<0,001$ & $\varphi=4,4 ; p<0,001$ \\
\hline
\end{tabular}

\section{Conclusions}

1. The purpose of this article was to describe the methods of organizing the educational process in order to develop semantic reading as a professionally significant quality in students of a defectological profile. 
2. The results of the work done confirm that the inclusion of specially selected tasks in the development of the discipline "Pedagogy" allows you to better prepare speech therapists in accordance with the standard of professional training in the context of informatization.

3. The digital lifestyle is gaining an irreversible character, so at the present stage there is a need to accept digitalization as an existing reality, it is necessary to find various opportunities in the digital space, focus on the specifics of this influence.

4. In the field of developing the skill of semantic reading, the importance of practiceoriented work to improve the speech culture of students is undoubted, which will allow, when used in self-study of digital educational resources (video clips, sound recordings, text documents), to consciously and meaningfully perceive them, which will make it possible to exchange diverse theoretical, methodological and practical resources, to contribute to the development of not only communicative, but also informational competence.

\section{References}

1. N. A. Kalugina, Pedagogical, informational and digital technologies in the professional training of future speech therapists (2019)

2. M. Méndez, Ten simple rules for developing good reading habits during graduate scho ol and beyond, 11 (2018)

3. V.V. Znakov, The Psychology of Understanding: Problems and Prospects (2005)

4. E. F. Tarasova, Research of speech thinking in psycholinguistics (1985)

5. T. A. Altukhova., I. N. Karachevtseva, Early diagnosis, prevention and correction of writing and reading disorders: materials of the 2nd Intern. conf. Grew up. assoc. dyslexia., 5 (2006)

6. A. R. Luria, Writing and Speaking: Neuro Linguistic Research (2002)

7. G. U. Soldatova, Digital generation of Russia: competence and safety (2017)

8. T. S. Komarova, Information and communication technologies in education (2012) 